\title{
Escuela Latinoamericana de Comunicación (ELACOM): referente histórico y conquista de la hegemonia en el pensamiento latinoamericano de la comunicación
}

\author{
Gustavo Adolfo León Duarte* \\ UNIVERSIDAD DE SONORA
}

De una década a esta parte, una de las iniciativas institucionales que quizás mejor simbolice la búsqueda de identidad en el Pensamiento Latinoamericano de la Comunicación (PLC) es el programa de trabajo que encarna la llamada Escuela Latinoamericana de la Comunicación (ецAсом). La voluntad de saber se centra en tratar de identificar una parte del tejido histórico referencial que le es propio a la construcción de la idea que representa la llamada ELACOM. La idea medular que se construye aquí, trata de colocar los antecedentes y referentes históricos de conocimiento desde la iniciativa y perspectiva conceptual y discursiva que le ha suministrado no solamente su procedencia y razón institucional, sino, además, en tratar de conocer el porqué de la ubicación en debate del programa de la ELACOM. El conjunto de recursos científicos heredados del PLC que existen hoy en estado objetivado, bajo la forma de instrumentos, de obras y de instituciones, así como en estado incorporado, bajo la forma de habitus científico, merecen sin duda un mayor esfuerzo para materializar y ampliar la iniciativa institucional de la ELACOM. No obstante, debe considerarse el más amplio consenso de participación de la comunidad académica latinoamericana de la comunicación.

* Profesor Investigador Titular en el Departamento de Ciencias de la Comunicación de la Universidad de Sonora, México. Es Doctor en Periodismo y Ciencias de la Comunicación por la Universidad Autónoma de Barcelona. El autor agradece las observaciones y los comentarios críticos realizados al presente documento por Miquel Rodrigo Alsina, Manuel Parès y Maicas, Miquel de Moragas i Spà, José Marques y Raúl Fuentes Navarro 
From the last decade to the present, one of the institutional initiatives which best symbolizes the search for identity in Latin American Thought on Communication (LATCO) is the work program symbolized and represented by the Latin American School of Communication (ELACOM). This study centers on identifying part of the referential historical framework that underlies the construction of the ideas represented by ELACOM. The main idea being constructed tries to place the historical background and references from the conceptual and discursive initiative and perspective that have contributed not only to its origin and institutional cause, but also in trying to understand the basis for the positioning of debate in the ELACOM program. The collection of scientific resources inherited by the Latin American thought on communication that exist today in an objetive state, under the form of instruments, works, and institutions, as well as in an incorporated state, under the form of scientific habitus, deserve without a doubt greater effort to materialize and expand the institutional initiative of ELACOM. Nevertheless, the broadest consensus of participation from the Latin American academic community in communication must be considered.

\section{Presentación}

Como producto de varias décadas de transitar entre luchas y conciliaciones por la legitimación del campo académico y el reconocimiento institucional, hoy los estudios de la comunicación en América Latina han acentuado la necesidad de una búsqueda de identificación y unidad en torno a ciertas propiedades que comporta la generación de conocimiento que le es propia a la comunidad latinoamericana de investigadores e investigadoras de la comunicación. Concretamente, entorno a la producción ética-epistemológica que despliegan no pocas fuentes conocidas y reconocidas al interior de la estructura del campo de estudios de la comunicación en América Latina. Sobre el terreno de la arena académica, el desarrollo histórico de la búsqueda de la identidad latinoamericana de la comunicación pasa por diversas guías bien documentadas que sirven de partida para ubicar y contextualizar el análisis de los debates dominantes sobre el tema. Una de las primeras guías implicaría retomar el hoy distante análisis planteado por Jesús Martín Barbero (1982) sobre la mencionada "relevancia social" del objeto de estudio en la investigación de la comunicación en América Latina. Significaría también, preguntarnos sobre qué problemas necesitan ser investigados y cuáles preguntas importantes deben ser hechas y atendidas en nuestros países latinoamericanos. (Martín Barbero, 1987; 1988). Sobre esta línea de análisis se tendría que explicitar, además, hasta qué punto y desde dónde 
están siendo renovadas o reconfiguradas aquellas "utopías fundacionales" de los estudios de la comunicación en Latinoamérica planteadas en diferentes etapas y momentos de desarrollo del campo por Luis Ramiro Beltrán (1974; 1978; 1982; 1999; 2004). También, en qué sentido se definen, en el caso hipotético de que así fuese, los términos del debate de la función social en la investigación de la comunicación planteado por Raúl Fuentes Navarro (1999), quien, por su parte, apunta que, pese a su gran importancia, esta es una discusión insuficientemente desarrollada por los investigadores latinoamericanos de la comunicación.

De una década a esta parte, una de las iniciativas institucionales que quizás mejor simboliza la búsqueda de identidad en el pensamiento latinoamericano de la comunicación es el programa de trabajo que simboliza y encarna la llamada Escuela Latinoamericana de la Comunicación (ELACOM). Al menos dos son las líneas que suponen la identificación básica de la producción investigativa de la hipotética ELACOM. Por un lado, la característica central de ser una singular investigación mestiza que incorpora diversas formas y corrientes de hibridación teóricas-metodológicas y, por otro, la permanente alusión renovadora de su posicionamiento ético-político (León, 2005). En tanto iniciativa y programa de trabajo institucional, la presencia y caracterización de la ELACOM ha sido discutida y cuestionada. El debate lo abrió el impulsor principal de la ELACOM, José Marques (1999; 1999a) en dos foros distintos en el mismo año de $1999^{1}$. En abril de ese año, en La Trinidad, Tlaxcala, México, en la conferencia inaugural del X Encuentro de la Asociación Mexicana de Investigadores de la Comunicación (AMIC), Marques planteó, a manera de propuesta, una revisión crítica de la experiencia brasileña como modelo para fortalecer la comunidad latinoamericana de la comunicación. Si bien la invitación fue recibida con bastante interés por los investigadores mexicanos, el debate central de su discusión se trasladó hasta el I Seminario Latinoamericano sobre la Investigación de la Comunicación, celebrado en Cochabamba, Bolivia, en noviembre de ese

${ }^{1}$ No obstante, habría que señalar que el profesor José Marques (1992, Pp. 5-25), sin duda uno de los investigadores más destacados y conspicuos dentro del campo de estudios de la comunicación en Brasil, inició desde tiempo antes un proceso de identificación y rescate del pensamiento de la ELACOM. Por ejemplo, en 1992, siendo presidente de la Asociación Latinoamericana de Investigadores de la Comunicación (ALAIC), mostró enfáticamente como una de sus principales preocupaciones el rescate urgente de la memoria del pensamiento "comunicacional" latinoamericano, centrándose en sus raíces contemporáneas, su proyección internacional y las tendencias emergentes que se cultivaban en el campo académico de la comunicación en América Latina.

Escuela Latinoamericana de Comunicación (ELACOM): referente histórico y conquista $\bullet 53$ de la hegemonia en el pensamiento latinoamericano de la comunicación 
mismo año. José Marques, Director de la cátedra unesco Comunicación en Brasil, consideró la existencia de lo que él llamó Escuela Latinoamericana de Comunicación, caracterizada por su mestizaje teórico, su hibridismo metodológico, su compromiso ético político y su dimensión extranacional.

Sin embargo, la discrepancia inmediata la asumió en el mismo foro Guillermo Orozco, impulsor de los estudios cualitativos sobre recepción de medios en México, al poner en duda la iniciativa de hablar de una Escuela, pues, dijo, conllevaría a algunas homogeneidades de las que la comunicología latinoamericana carece. Orozco respaldó, en cambio, otras alternativas, también aportadas por Marques, como la Vía Latinoamericana de Comunicación (Torrico, 2000). Argumentó, además, que quizás en esta región se empiece a configurar una Escuela Latinoamericana de Comunicación en torno a la propuesta de Jesús Martín Barbero, quien ha revolucionado los abordajes teóricos metodológicos de los procesos comunicativos a partir de los medios y las mediaciones.

Desde 1999 hasta el último foro abierto del Coloquio Internacional de Estudio sobre la Escuela latinoamericana de la Comunicación (CELACOM) ${ }^{2}$ José Marques (2004; 2004a; 2004b; 2005; 2005a) ha articulado un discurso renovador entorno a la ELACOM. Entre otras cuestiones centrales, ha definido las metas propuestas para el itinerario del debate que debe centrar el mapa del pensamiento y del investigador latinoamericano de la comunicación. Según Marques (2004, pp. 210-211), estas propuestas deben centrarse en tres aspectos generales: la naturaleza del proceso de la comunicación, la autonomía teórica y el fomento a la crítica metodológica y, por último, el rescate del conocimiento empírico en su triple dimensión: autóctono, mestizo y popular.

En relación al primer aspecto, la naturaleza del proceso de la comunicación debe iniciar y contemplarse en su análisis comunicativo como un desarrollo

${ }^{2}$ Los CELACOM son una iniciativa institucional promovida y auspiciada por la Cátedra UNESCO Comunicación para el Desarrolla Regional en Brasil y, desde luego, por la universidad que les cobija: la Universidad Metodista de Sáo Paulo (UMESP). La orientación de los eventos se realizan bajo la orientación de su director titular, el Profesor Dr. José Marques. Con una periodicidad anual, el Primer Coloquio Internacional de Estudios sobre la Escuela Latinoamericana de Comunicación se llevó a cabo en Sao Paulo, Brasil, entre el 24 y 26 de marzo de 1997; este primer Coloquio fue dedicado a las ideas comunicacionales del investigador y Profesor boliviano Dr. Luís Ramiro Beltrán. En este evento, como en cada uno de los siete coloquios posteriores efectuados a la fecha, se publicaron las contribuciones o trabajos de investigación recibidos bajo el título A Trajetória Comunicacional de Luiz Ramiro Beltrán; con este volumen se inicia la publicación unEsCo-Umesp titulada "Serie Análisis de la Escuela Latinoamericana de la Comunicación". 
social amplio. Para ello, se debe estimular la realización de estudios sobre el comportamiento de los emisores (mapas etnográficos o sociográficos) y sobre los efectos (impactos psicosociales, políticos, educativos), con la finalidad de influir en la etapa de construcción de nuevos productos mediáticos y de producir conocimiento aplicable, o bien hacer la crítica socialmente utilitaria. Con respecto a la autonomía teórica y el fomento de la crítica metodológica, se trata aquí, de acuerdo con el análisis planteado por Marques, de recurrir a estrategias de investigación que permitan mejorar la calidad de los productos mediáticos demandados por la sociedad. Ello debe ser fruto de un diálogo abierto y equilibrado con las ciencias sociales, superando complejos de inferioridad intelectual que fragilizan al sector más diletante de nuestra comunidad. Finalmente, el tercer aspecto del itinerario que define Marques, es la apuesta por el rescate de un tipo de conocimiento de saber práctico, en su triple dimensión: autóctono, mestizo y popular. Marques apunta que, en gran medida, la tradición "comunicacional" latinoamericana proviene de adaptaciones metodológicas que se hicieron de modelos importados (estadounidenses y europeos) y de soluciones ingeniosas que asimilamos de las culturas populares. ${ }^{3}$ En la formación de las

${ }^{3}$ Efectivamente, para el caso de América Latina, se ha podido comprobar que los procedimientos de síntesis entre la metodología norteamericana y la reflexión europea vienen marcados por las tendencias epistemológicas y los modelos metodológicos foráneos y externos a la investigación propiamente latinoamericana (León, 2006, pp. 9-85). Por un lado, aquella tendencia empírica funcionalista que predominó desde 1950 y hasta fines de la década de los años setenta, la Mass Communication Research. Por otro, las tendencias originarias de Europa, donde destacan la perspectiva de la teoría crítica (que a partir de 1964, con la publicación en Estados Unidos de El hombre unidimensional de Herbert Marcuse se le empieza a nombrar e identificar en América Latina como la Escuela de Frankfurt, lo mismo que algunos de sus más destacados representantes como Adorno, Horkheimer, Benjamin y el propio Marcuse) y las diferentes interpretaciones marxistas: la semiótica de Pierce y el estructuralismo francés. En esta última corriente de investigación cobra una importancia determinante el grupo del Centro de Investigaciones Sociales del Instituto Torcuato Di Tella, en Argentina, bajo el liderazgo de Eliseo Verón (1968; 1969; 1970) y Luís Prieto (1967; 1975; 1978), cuya preocupación por la interpretación ideológica de los medios y su esfuerzo por conseguir una síntesis teórica entre psicoanálisis, marxismo y lingüística estructural, hallarían una amplia repercusión en toda América Latina a partir de la década de los años setenta (Moragas, 1982). La vitalidad del estructuralismo, unida a la capacidad de Verón para formar cuerpos académicos y de investigación en Buenos Aires, llevan a este investigador, en octubre de 1970, a formar y dirigir la Asociación Argentina de Semiótica, sólo un año después de fundarse la Asociación Internacional de Semiótica. Cuatro años más tarde fundaría la primera revista especializada en semiótica y una de las primeras revistas especializadas en Latinoamérica: Lenguajes.

Escuela Latinoamericana de Comunicación (ELACOM): referente histórico y conquista $\bullet 55$ de la hegemonia en el pensamiento latinoamericano de la comunicación 
nuevas generaciones de comunicadores, José Marques propone recurrir al arsenal empírico guardado por las corporaciones profesionales, ayudar a sistematizarlo y actualizarlo a partir de la referencia crítica que siempre caracterizó el trabajo universitario.

Pero, lo anterior también provee y lleva, casi de manera ineludible, al planteamiento de varias preguntas de investigación que sirven, además, para configurar el marco de abordaje del tema y objeto de estudio: ${ }^{4}$ ¿Qué es la Escuela Latinoamericana de la Comunicación? ¿Qué características específicas soportan a la tesis de existencia de la ELACOM? ¿Por qué este proyecto cobra cada año una mayor importancia en el campo académico de la comunicación en Latinoamérica? ¿Cómo opera su programa de trabajo? ¿Existe acaso algún apoyo institucional que respalde el programa de trabajo de la ELACOM? Si es así, ¿Cuál institución es y en qué consiste dicho apoyo? ¿Qué problemas presenta la idea y el programa de trabajo de la ELACOM entre las fuentes del campo académico de la comunicación de Latinoamérica? O, por el contrario, ¿Qué problemas se presentan en el campo académico de la comunicación de Latinoamérica para consolidar el programa y las perspectivas de trabajo de la ЕLACOM?

En este contexto, el objetivo del presente estudio es identificar una parte del tejido histórico referencial que le es propio a la construcción de la idea que simboliza la llamada ЕLACOM. La idea medular que se construye aquí, trata de colocar los antecedentes y referentes históricos de conocimiento desde la iniciativa y perspectiva conceptual y discursiva que le ha suministrado no solamente su procedencia y razón institucional, sino, además, en tratar de conocer el porqué de la ubicación en debate del programa de la Escuela Latinoamericana de la Comunicación. Dicha ubicación del objeto se realiza desde una perspectiva de análisis que contiene dos planos relativamente diferenciados. Por un lado, desde la perspectiva de algunas ilustres y destacadas posiciones o fuentes del campo académico de la comunicación en nuestra región y, concretamente, desde la perspectiva del creador de la tesis de la existencia de la ELACOM, el Profesor Dr. José Marques. Por otra, desde las condiciones y representaciones que le son propias a las instituciones, en este caso tanto a la UNESCO como a la Universidad Metodista de São Paulo (UMESP), a través de la Cátedra unEsco

${ }^{4}$ Las preguntas de investigación son entendidas aquí como planteamientos de apoyo a las guías del estudio y a los objetivos concretos de investigación. Plantear el objeto y problema de estudio a través de preguntas tiene la ventaja de presentarlos de manera directa, minimizando la distorsión y, sobre todo, apuntar hacia lo que habrá de hacer sentido y centrar nuestra voluntad de saber en el estudio. 
Comunicación para el Desarrolla Regional en Brasil, cuyo Director Titular es precisamente el Profesor José Marques. Desde ambas perspectivas de análisis se trata de ir descifrando y reconstruyendo un enigmático "rompecabezas" llamado elaCom. Se trata, de ir trazando, entre documentos e ideas separadas por al menos cuarenta años, una idea clara a cerca de su definición y su origen histórico. De ir marcando una delimitación a las posibles características que le son propias, como de puntualizar aquellos problemas que enfrenta en tanto se conforma como un proyecto y a la vez como un programa institucional de trabajo hasta un punto concreto en el tiempo: el desarrollo institucional que ha alcanzado hasta marzo de 2005, es decir, hasta la realización del octavo CELACOM. El desarrollo de contenidos se efectúa a partir de un análisis interpretativo de carácter histórico hecho a diversas producciones sobre el tema y objeto de estudio. ${ }^{5}$ En el presente documento, por motivos de espacio, el enfoque se centra fundamentalmente en una parte importante de la producción discursiva y la práctica científica (tratada aquí como un proceso histórico de conocimiento y reconocimiento de sus agentes e instituciones) originada por su principal impulsor, José Marques. El periodo de estudio abarcó desde mediados de la década de los sesenta hasta 2005.

\section{Elacom. Cepas y procedencias institucionales}

Un camino que nos revela ciertas pistas sobre el desarrollo de los orígenes que soportan tanto la iniciativa como el programa de trabajo de la ELACOM tiene un precedente no sólo cercano a lo que históricamente ha venido aconteciendo en el discurso académico de los estudios de la comunicación en Latinoamérica. Todo parece indicar que una de las ideas que soportan el proyecto de la ELACOM, creado e impulsado por el Profesor José Marques, tiene sus cepas y procedencias en el campo académico de la comunicación en Brasil. Concretamente en aquellas ideas que durante la década de los 70 inspiraron, por un lado, algunas prácticas en la enseñanza y la investigación de la comunicación en este país y, por otro, la relación de dichas prácticas con el trabajo académico

${ }^{5}$ Dichos análisis devienen de una propuesta metodológica más amplia que hemos incorporado en trabajos recientes (León, 2005, 2006, 2006a). Por nombre se distingue como Metodología de Identificación de las Prácticas Institucionales para el Campo Científico de la Comunicación (MIPICC). El objetivo de la estrategia metodológica es lograr el acopio de indicadores y categorías de información que permitan analizar el contexto institucional del campo académico de la comunicación en América Latina.

Escuela Latinoamericana de Comunicación (ELACOM): referente histórico y conquista 57 de la hegemonia en el pensamiento latinoamericano de la comunicación 
por demás arduo y resistente que promovieron algunos centros y asociaciones dedicadas a la enseñanza y la investigación de la comunicación en Brasil. El propósito de ambos cruces de ideas no fue otro que el consolidar las bases del proceso de institucionalización de los estudios de la comunicación, iniciado durante la década de los 40 al instalarse formalmente la Escuela de Periodismo Cásper Libero en São Paulo, Brasil. Dichas prácticas pueden ser claramente reconocidas en Marques, Anamaria Fadul y Carlos Lins da Silva (1979, pp. 5-294), al analizar la crisis de la enseñanza y la investigación en Brasil en la década de los años 70.

Por ejemplo, una primera característica que puede ser reconocida como central en la identificación de un conocimiento comunicativo propio en la región es el eje de la no-dependencia de modelos foráneos de comunicación. Este primer elemento es ya familiar en las prácticas discursivas de Marques (1978; 1979) al resaltar el hecho de que ciespal fue uno de los pocos centros de investigación que encarnó un proyecto en defensa de los valores culturales nacionales en América Latina. El Profesor Marques apunta, en un primer momento, el papel institucional que desarrolló el Centro Internacional de Estudios Superiores de Periodismo para Amerita Latina (CIESPAL) en los procesos de formación docente y de investigadores durante las décadas de los ańos 60 y 70 . Con una conciencia basada en la "inadecuación” de modelos teóricos-metodológicos foráneos a las situaciones propias de la vida brasilera y latinoamericana emergerían lenta y gradualmente caminos alternativos para superar la dependencia. Situado en el análisis del contexto urbano industrial de principios de los 70 en Brasil, Marques (1979, p. 37) afirma que si bien CIESPAL en un principio se vinculó a "la capacitación de la mano de obra intelectual que era necesaria para justificar y hacer funcionar los equipamientos y los estilos de vida importados de los países metropolitanos", dicha situación cambió y se reorientó hacía una política latinoamericanista, no-dependiente; de rechazo a la hegemonía de los países industrializados y defensa de los valores culturales nacionales de la región; en contra de la "penetración de estereotipos consumistas y alienadores de las naciones desarrolladas".

Por otro lado, Marques (1979, p.38) reconoce también que la enseñanza de la comunicación en Brasil durante los años 70 presentaba un diagnóstico extremadamente complejo y aceptaba, como premisa central, que los problemas en la enseñanza de la comunicación no solamente eran problemas aislados a la realidad brasileña sino que presentaban los mismos síntomas de malestar del inadecuado sistema de enseñanza superior entonces vigente en ese país. En- 
tendía, entonces, que la estructura de una escuela de comunicación no podía circunscribirse a la esfera de la enseñanza sino que tenía que ampliarse a actividades de auténtica práctica profesional, sustentadas en un trabajo continuo de investigación capaz de influir positivamente en la operación del sistema nacional de comunicación. Marques (1979, p.38) sentenciaba que solamente cuando se construyan "escuelas de esta naturaleza, se acelerará la tecnología nacional y se romperá la dependencia que nos atrae inevitablemente al carro de los países desarrollados".

Por su parte, Anamaria Fadul (1979, pp. 50-57), destacaba que no solamente la adecuada vinculación entre teoría y práctica podía ser pensada en función de la propia relación entre escuela y sociedad sino también por la superación de dos factores centrales en la relación de acción pedagógica y escuelas de comunicación en Brasil: por un lado, en la superación de la alta dependencia nacional que presentan las escuelas respecto de la bibliografía extranjera en comunicación; por otro, en la no-aceptación de que en ningún curso de comunicación se propusiese como objetivo la formación de sujetos con posibilidades de acción en la sociedad brasilera. Para Fadul (1979, p.57), la teoría de la comunicación debería centrar la investigación en este sector pero jamás podía ser pensada como una teoría general válida para todos los hombres y para todos los países, sino como una teoría que permitiese entender de manera particular el fenómeno de la comunicación en la región y concretamente en Brasil. Es por ello que, para Fadul (1979, p.58), enfrentar la crisis de la enseñanza y la investigación en el Brasil de los 70 significaba en primer término aceptar como necesaria la reorientación de la enseñanza de la comunicación a los términos y propuestas de la Asociación Brasileña de Enseñanza e Investigación de la Comunicación (ABEPEC) y a la regulación misma de la enseñanza de la comunicación social en Brasil, misma que había sido promulgada en mayo de 1942 a instancias de reivindicar las aspiraciones que desde principios de siglo entrañaban y exigían los y las periodistas brasileños.

\section{Abepec e Intercom. Primeras disposiciones institucionales DE LA ELACOM}

La ABEPEC surgió en 1975 como producto de la primera tentativa de aglutinar a los líderes de la enseñanza y la investigación en las escuelas de comunicación en Brasil en virtud de que existía la necesidad de enfrentar colectivamente la crisis de calidad en la enseñanza y de estimular la investigación (Marques, 1991, pp.

Escuela Latinoamericana de Comunicación (ELACOM): referente histórico y conquista 59 de la hegemonia en el pensamiento latinoamericano de la comunicación 
130-131). De acuerdo con la justificación de la propuesta que realizó la ABEPEC en Brasilia, la última semana de mayo de 1976, un primer reconocimiento que es válido para denotar los términos de las recomendaciones y propuestas para la reorientación de la enseñanza de la comunicación es conocer la evolución de la enseñanza de la comunicación en Brasil entre 1942 y 1977. Concretamente, los datos ofrecidos por la ABEPEC (Marques, Fadul y Lins da Silva, 1979, pp. 75-77), en este período, muestran que Brasil presenta diversas características predominantes en la enseñanza de la comunicación. Dichas características, en términos generales, se ubican en tres fases o etapas comparativamente diferenciadas: una primera fase es la que la ABEPEC define como "Clásico-Humanista” y que trascurre desde el inicio de la regulación de la enseñanza (1947-1948) hasta la segunda mitad de la década de los años 60. La característica predominante de la enseñanza en el periodo es la orientación europea clásica, concentrada casi exclusivamente en el periodismo gráfico; su orientación básica estuvo conformada por actividades y aspectos de corte literario, ético-jurídico e histórico. La segunda fase, denominada "Científico-Técnica", inicia la segunda mitad de la década de los 60 y se consolida al final de la misma, inmediatamente después de la implantación del currículo de la enseñanza de la comunicación en 1969. En este periodo, reincide el tratamiento científico-empírico y técnico del fenómeno comunicativo. En el ámbito curricular son introducidas materias que están dirigidas a la enseñanza de los fundamentos psicológicos, sociológicos y antropológicos de la comunicación y sus técnicas de investigación donde sobresalían el análisis de contenido y el análisis de base cuantitativo. Además, en esta fase se conserva la orientación "gutemberguiana" al conferirle un excesivo peso a las materias destinadas a la preparación de profesionales para trabajar en medios impresos. Finalmente, la tercera fase es la que ABEPEC apela con el título de "crítico-reflexiva". Su periodo transcurre desde mediados de la década de los años 70, justo después de que los estudios de licenciatura pierden el carácter de terminal tras el establecimiento de la política de post-graduación implementada por el gobierno federal. La característica principal del periodo es que las escuelas han comenzado a adquirir las condiciones necesarias de tranquilidad y, con ello, el desarrollo de la reflexión, como pasos previos para sentar las bases de su institucionalización, acción que no se concretaría hasta la década de los años 80 .

Según la visión de la ABEPEC (Marques, Fadul y Lins da Silva, 1979, pp. 77 85), una de las principales fallas que comporta la enseñanza de la comunicación en Brasil es que no se enfocaba a conocer ni a solucionar los problemas de la 
propia realidad. La reorientación propuesta por la ABEPEC partía de que tanto la realidad regional como la brasilera comportaban características propias, que sometidas a través de un estudio comprensivo se podía llegar a proponer soluciones adecuadas para los problemas. Así, la ABEPEC propondría un currículo conformado por un amplio cuadro de materias y referencias que se traduciría en el análisis práctico de la propia producción cultural brasileña. Además, se rompería la tendencia a formar "profesionales capacitados para la manipulación tecnológica" y se relevaría por un modelo "orientado en la intervención social para el desarrollo" en función de entender que la "comunicación es indispensable para mejorar la calidad de vida en tanto afecta a la productividad, a la expresión personal como a las necesidades de vínculos sociales". Sin embargo, para ABEPEC la estructura sugerida para un currículo mínimo en las escuelas de enseñanza de la comunicación en Brasil no debía parar ahí. La ABEPEC propondría una nueva y completa consideración respecto al carácter de estudio de la comunicación. En primer término, propondría entender a la comunicación como un proceso, en la medida en que se estudia sincrónica y diacrónicamente los fenómenos de comunicación en términos de la variedad de operaciones que la integran. Segundo, estudiar a la comunicación como un sistema, en la medida en que los procesos de comunicación no son independientes de las estructuras en que operan u ocurren. Tercero, estudiar la comunicación como arte, en la medida en que los procesos de elaboración y transmisión de mensajes son envueltos bajo complejos mecanismos de creación aún cuando algunos mecanismos son bien conocidos y sistematizados. Cuarto, se propondría estudiar a la comunicación como ciencia, en la medida en que su estudio constituye una reflexión sobre principios, métodos y datos que permiten el tratamiento conceptual y explicaciones a los fenómenos de comunicación.

Sin embargo, y de acuerdo con las pistas localizadas, los primeros elementos contextuales históricos relacionados con la conformación y creación de la ELACOM por parte de las fuentes de campo, nos indican que el debate del estudio, la enseñanza y la investigación de la comunicación en Brasil pronto dejó el panorama nacional. Al crearse la Sociedad Brasileña de Estudios Interdisciplinares de la Comunicación (INTERCOM), y, concretamente, los encuentros llamados Ciclos de Estudos Interdisciplinares da Comunicação, se destaca un cambio notable en el paisaje y la perspectiva de análisis. ${ }^{6}$ Con ella, las representaciones

${ }^{6}$ La InTERCom es una sociedad civil fundada en diciembre de 1977 en São Paulo por académicos, investigadores e intelectuales brasileros que pensando, practicando e investigando

Escuela Latinoamericana de Comunicación (ELACOM): referente histórico y conquista $\bullet 61$ de la hegemonia en el pensamiento latinoamericano de la comunicación 
simbólicas de la ELACOM cobraran, sin lugar a dudas, sus primeras señales de vida. La internacionalización de las discusiones sobre la enseñanza y la investigación de la comunicación ocurren en dos planos y momentos distintos, de acuerdo con el propio Marques (1983, pp. 7-13): primero, a través de la reflexión que investigadores brasileros realizaran sobre las tendencias de la investigación en comunicación en países desarrollados; segundo, a través del intercambio y la participación directa que investigadores europeos, norteamericanos y latinoamericanos realizaron y contribuyeron para revisar críticamente los modos de investigar los procesos de comunicación e interacción simbólica en los países de origen. En este sentido, Marques (1983, p.11), apunta un elemento que aquí consideramos como una segunda característica que es medular para ir conformando un conocimiento comunicativo propio en la región y que se produce en el intercambio de pares internacionales a través del Ciclo INTERCOM de 1982: el sepultar un antiguo "complejo de inferioridad" que siempre acompañó a los investigadores brasileros de sus pares extranjeros. Para Marques este evento situó en un plano de igualdad a los investigadores brasileños y estableció un nuevo orden de relación entre investigadores de diferentes países al grado de "romperse los parámetros peculiares de relaciones de subordinación entre las naciones en el plano internacional".

la comunicación, no disponían de un espacio propio donde pudiesen debatir críticamente sobre la comunicación contemporánea (Marques, 1983, pp. 237). INTERCOM surge con dos características bien nítidas: la interdisciplinariedad y el pluralismo, de ahí que, además de la integración de investigadores y estudiosos de la comunicación, se vincularan también diversos científicos sociales que privilegian a la comunicación en cuanto objeto de análisis profesional y académico. Es un hecho que los títulos de las temáticas analizadas en los cinco primeros encuentros o ciclos de InTERCOM pueden ser un fiel reflejo del cambio de panorama al que hacemos mención. Es evidente que el plano nacional abre paso al plano regional latinoamericano. Los títulos de los Ciclos INTERCOM entre 1978 y 1982 son: 1978) Estrategias para o Encino de Comunicação; 1979) Modas de Comunicação das Clases Subalternas; 1980) Estado, Populismo e Comunicação no Brasil; 1981) Comunicação, Hegemonia e Contra-informação; 1982) Teoria e Pesquisa em Comunicação. Panorama Latino-Americano.

${ }^{7}$ El ciclo inTERCOM de 1982 también reveló para Marques (1983, p. 11) un tercer elemento que a la postre (justamente una década después) sería clave para unir a la comunidad académica latinoamericana: la manifestación de una nueva fase de investigación en Brasil (que de hecho fue un elemento homogéneo en gran parte de Latinoamérica) al surgir investigadores jóvenes que incursionarían al campo académico de este país y que, bajo ningún tipo de relación subalterna establecida, se lanzaron al debate aportando contribuciones valiosas y empujando de la investigación participativa y de la investigación denuncia a la investigación acción. 
Un vínculo importante se teje entre las pistas de estudio que significan el "complejo de inferioridad" y el eje de la "no dependencia teórica": el llamado "combate al aislacionismo". De hecho, en esta incursión histórica se ha podido comprobar que el llamado "combate al aislacionismo", en tanto característica contextual significativa en la tesis sobre la ELACOM, guarda una estrecha relación con el contexto referencial de la formación de Marques. Pero, también, con un planteamiento futuro que caracterizará la posición de José Marques al ser el primer académico latinoamericano en plantear la tesis de la existencia de la Escuela Latinoamericana de la Comunicación: el llamado "síndrome del colonizado", tratado más adelante.

Icinform, Alaic y Elacom. Tres proyectos, un impulsor protagónico

El "combate al aislacionismo", tercera característica central que apoya la tesis de la existencia de la ELACOM, deviene de la marca que define a una institución pionera en la investigación de la comunicación en Brasil y Latinoamérica: el Instituto de Ciencias de la Información (ICINFORM). El ICINFORM surge en la Universidad Católica de Pernambuco (en la región noreste de Brasil) en 1963, bajo el liderazgo intelectual del Profesor Luiz Beltrão. El Profesor José Marques, entonces incursionando al campo de la comunicación en Brasil, se integró al equipo coordinador del ICINFORM, por lo que se vio fuertemente vinculado, sino influenciado, en y por las filosofías y las políticas de trabajo del Instituto y de su Director, Luiz Beltrão. ${ }^{8}$ En el contexto de los estudios de la comunicación en Brasil de mediados de la década de los 60, el ICINFORM implementaba estrategias de desarrollo que se orientaban más por el estimular la importancia de la investigación, el espíritu investigativo y el valor y la pertinencia de su práctica, antes que el "puro rigor científico" que ello implicaba (Nobrega, 2000, p. 162). Uno de los modos o formas que enfatizó Beltrão para combatir la falta de participación en el quehacer de la investigación por parte de la comunidad académica en Brasil, fue el implementar una "visión amplia del mundo" en la formación de los periodistas (hasta entonces no se manejaba el término comunicólogo o comunicador). Dicha visión puede ser claramente registrada en el I Curso de Ciencias de la Información, que se realizó en Recife, Pernambuco, Brasil, entre el 16 de enero y el 4 de marzo de 1965. En este I Curso, pionero en su tipo

${ }^{8}$ Guías de estudio en esta dirección son desarrolladas en Nóbrega (2000), Targino (2000) y Gobbi (2001).

Escuela Latinoamericana de Comunicación (ELACOM): referente histórico y conquista $\bullet 63$ de la hegemonia en el pensamiento latinoamericano de la comunicación 
en Brasil y en América Latina, se concretaría el llamado que Beltrão haría a la comunidad académica brasilera y latinoamericana para integrar y aumentar su participación en el campo de estudios bajo el lema "combate al aislacionismo". Por añadidura, el "combate al aislacionismo", junto con la presentación de la característica de "no dependencia teórica-metodológica" y el derrumbamiento de "los complejos de inferioridad", la entrada en escena de jóvenes investigadores en la comunidad académica de la comunicación en Brasil y en Latinoamérica implicó, entre otras cosas, el inicio de un proceso de revitalización y empuje de los mecanismos de auto sustentación y retroalimentación de la ALAIC, cuya estrategia de participación, al iniciar la década de los 80, estaba pensada en pasar de las fronteras nacionales y de los particularismos regionales (Marques, 1992, p.7). En nuestra opinión con la explotación "productiva" de dichas características, se consolidaría implícitamente el desarrollo gradual seguido por la investigación latinoamericana de la comunicación y se demarcarían aquellos caminos alternativos identificados con el mestizaje teórico y la práctica de un sincretismo metodológico.

La ALAIC tuvo una actuación de altibajos y claroscuros desde inicios de los años 80 y hasta su reconstitución en 1989. Desde mediados de la década de los 60 hasta prácticamente el cierre de la década de los 80 , el periodo fue atravesado por varios elementos importantes de carácter coyuntural, desde la época de la distensión (1962-1975) y el inicio y desarrollo de un nuevo periodo de recrudecimiento de la guerra fría (1975-1989), hasta el lanzamiento de la campaña y búsqueda de un nuevo orden internacional de la información y la comunicación. ${ }^{9}$ Bajo este contexto, no fue extraño que a lo largo y ancho del

${ }^{9} \mathrm{El}$ lanzamiento de la campańa inicia con la declaración de un nuevo orden económico internacional adoptada por consenso el 1 de mayo de 1974, durante la VI Asamblea Especial de la ONU; las disposiciones adoptadas aquí se concretarían en 1978 durante la XX Conferencia General de la unesco con la presentación de los primeros resultados del Informe de la Comisión McBride, el cual se estructuró en función de dos conceptos antagónicos: Nuevo Orden Internacional de la Información y la Comunicación (NOMIC) y el libre flujo de la información (Free Flow of Information). A fines de la década de los años 70, de los 4.600 millones de hombres y mujeres que vivían en 135 países de este planeta, menos de mil millones y medio tenían derecho a comprar un periódico "libre" y voluntariamente; en el llamado "tercer mundo" (hasta entonces, 82 países que totalizaban aproximadamente 1.300 millones), se agregaba al control riguroso de la información y la censura, el hecho de que las actividades de recogida, elaboración y difusión de las noticias casi en tu totalidad provendría de agencias noticiosas internacionales como la AP y UPI (de Estados Unidos de América), APF (de Francia) y la agencia Reuters (del Reino Unido), que ignoraban, y 
subcontinente aparecieran centros e institutos de investigación que, organizados como entidades de interés público pero gestionados por el sistema de la administración privada, abordaran los procesos y fenómenos comunicativos desde diferentes perspectivas de análisis macro-político hasta el plano microideológico; de hecho, las tendencias hegemónicas en la investigación de la comunicación eran apoyadas y subsidiadas para su financiación por recursos provenientes principalmente de agencias y organizaciones extranjeras -no latinoamericanas- como la unEsco, las Fundaciones Ford, Frederic Ebert, Konrad Adenauer y otras.

Elacom. Generaciones de intelectuales e identidades multifacéticas

$\mathrm{Al}$ ingresar la gran mayoría de las universidades latinoamericanas tardíamente al escenario de la investigación de la comunicación, las modernas facultades de comunicación que se levantaban en las principales metrópolis latinoamericanas a principios de la década de los 70 no siempre significaron, como sucede hasta hoy en día en una gran parte de las universidades públicas de la región, un compromiso con la producción de conocimiento. De ahí, que la generación de conocimiento comunicativo (y no comunicativo) en los centros e institutos de comunicación que germinaban en la región alcanzaron una fuerte resonancia, en un periodo, reiteramos, que se caracterizaba por la búsqueda de alternativas comunicacionales y por la construcción de políticas democráticas de gestión de los medios masivos. Desde la teología de la liberación, la denuncia del imperialismo cultural, las corrientes estructuralistas, "frankfurtianas" y marxistas e inclusive los postulados heredados del funcionalismo norteamericano, se hicieron presentes, e impregnaron en mayor o menor medida, el pensamiento comunicacional latinoamericano tanto en el aula como en la configuración del campo académico de la investigación.

A mediados de la década de los 80 , se manifiesta un claro declive de los centros e institutos de investigación. Ello ocurría en función de la crisis económica que hundía tanto a la unEsCO como a la mayoría de las agencias internacionales de financiación, influenciadas, además, por el clima de polarización

continuarían ignorando, gravemente los intereses de los países en "vías" de desarrollo. Para mayor información, se puede consultar: El Pais, 04/04/1980: VII, en la siguiente dirección electrónica: http://www.portalcomunicacion.com/macbride/esp/mb_1_b.htmll (dirección consultada el día 17.12.2004).

Escuela Latinoamericana de Comunicación (ELACOM): referente histórico y conquista $\bullet 65$ de la hegemonia en el pensamiento latinoamericano de la comunicación 
ideológica que confluía, paradójicamente, entre la Perestroika de Gorbachov y la caída del Muro de Berlín. Según la visión de Marques, el sentimiento de derrota política que asumió la izquierda latinoamericana por ello, se fraccionó en luchas electorales por la democracia pero también debilitó el campo de la comunicación en la región: "En varios países de la región, eso influyó para la desmovilización de las instituciones, la última de las cuáles en desaparecer fue el IPAL..." (Marques, 2001, p.21). A pesar de ello, las bases de la reflexión critica sobre los fenómenos generados por la acción y el impacto de los medios en las sociedades latinoamericanas estaba ya consolidada. De acuerdo con Marques (2001, p.23), la identidad propia del pensamiento latinoamericano de la comunicación se inspiró en dos matrices ideológicas convergentes que fueron desarrolladas durante la década de los 60: La teoría de la dependencia y la teología de la liberación. Algunos estudiosos que son representantes del período embrionario en el estudio de la comunicación en América Latina y a los que Marques (2001, p.24), llama "pioneros" son: Antonio Pasquali, Luís Ramiro Beltrán, Luiz Beltrão, Paulo Freire, Jorge Fernández, Eliseo Verón, Roque Faraone, entre otros, los cuales lanzaron "tesis originales" y "construcciones científicas autóctonas" en el estudio de los fenómenos comunicacionales.

Sin embargo, para Marques (2001, p.24), la mayor proyección de los estudios de la comunicación en América Latina ocurre durante la década del 70 , justo con la participación de una siguiente generación de estudiosos de la comunicación a la cual ha denominado "grupo de innovadores", pues, según este enfoque analítico, son los responsables del avance metodológico de los estudios comunicacionales al incorporar modelos analíticos probados en las ciencias de lenguaje y las ciencias sociales en general. De acuerdo con Marques, a esta generación pertenecen Armand Mattelart, Heriberto Muraro, Jesús Martín Barbero, Anamaria Fadul, Mario Kaplún, Juan Díaz Bordenave, Fátima Fernández, Eleazar Díaz Rangel, entre otros. Para la década de los ochenta se concretaría lo que Marques (2001, p.25) denomina "grupo de renovadores" en la ELACOM. Se trata aquí de un grupo nutrido de intelectuales que, ubicados en facultades de comunicación de diversas universidades de la región, tuvo la capacidad de comprensión y explicación del pensamiento latinoamericano sobre medios, estructuras de poder, mediaciones culturales, usos y gratificaciones sociales, sus potencialidades educacionales, etc. En este grupo sobresaldrían algunos investigadores e investigadoras como Marcelino Bisbal, Carlos Eduardo Lins da Silva, Jorge González, Raúl Fuentes Navarro, Guillermo Orozco, Raúl Trejo, Patricia Terrero, Valerio Fuenzalida, Tereza Quirós, entre otros. 
El primer encuentro formal de estas tres generaciones que abarcan, según la posición de José Marques (2001, p.25) las décadas del 60, 70 y 80, fue el I Congreso Latinoamericano de Ciencias de la Comunicación que se llevó a cabo en São Paulo, Brasil, durante la segunda semana de agosto de 1992. Fue en este foro, donde las tres corrientes intercambiarían sus puntos de vista y conexiones con una nueva generación que asume el liderazgo en la comunidad académica de los estudios de la comunicación en América Latina. Es en este momento donde, según el profesor Marques (2001, p.26), el campo de la comunicación en Latinoamérica se robustecería en una doble fase: por un lado, porque la Escuela Latinoamericana de la Comunicación, en cuanto corriente de pensamiento, estaría confirmando su vitalidad a través de la emergencia de una nueva generación intelectual que Marques llamaría provisionalmente como "continuadores" y, por otro, porque la Escuela Latinoamericana de la Comunicación estaría concluyendo su trayectoria histórica al abandonar aquel perfil original de "extra-nacional", para asumir una "identidad multifacética", en torno a una región estructurada por "comunidades nacionales" de pensadores comunicacionales.

\section{ELACOM, TRAS LA CONQUISTA DE LA HEGEMONÍA EN EL PENSAMIENTO} LATINOAMERICANO DE LA COMUNICACIÓN

Para empezar a definir lo que por nombre lleva Escuela Latinoamericana de la Comunicación, habría primero que señalar que los significados explícitos a la ELACOM son prácticamente inexistentes. De hecho, el único señalamiento indirecto encontrado al respecto es aquel que María Cristina Gobbi (2001, p.12) apunta respecto a "la definición de la ELACOM que ha hecho Marques aludiendo a un grupo de investigadores que piensan los fenómenos de comunicación relacionados con la cultura latinoamericana”. De acuerdo con Gobbi, el profesor Marques ha definido las características singulares de la ELACOM, fundamentalmente a partir de la práctica del hibridismo y el mestizaje. Como ya anotábamos, desde principios de la década de los noventa, el Profesor Marques se manifestaba como la primera fuente de campo que identificaba la existencia de lo que él llama la Escuela Latinoamericana de la Comunicación. Hasta 1992, Marques (1992, pp. 5-25), identificaba varias peculiaridades en torno a la producción de conocimiento comunicativo en Latinoamérica. Entre otros elementos y características importantes de la ELACOM, Marques reconocía, a partir de la posición de varios investigadores(as) conocidos(as) y reconocidos(as) en los estudios de la comunicación en el mundo, las siguientes peculiaridades:

Escuela Latinoamericana de Comunicación (ELACOM): referente histórico y conquista $\bullet 67$ de la hegemonia en el pensamiento latinoamericano de la comunicación 
a) Un modelo de estudio de la comunicación propio del contexto y las necesidades históricas latinoamericanas, donde no domina ni la corriente empírica norteamericana ni la reflexión crítica europea; b) Un modelo propio fuertemente marcado por las implicaciones políticas de la investigación y la reflexión crítica sobre la comunicación de masas, que durante las décadas de los 70 y 80 llegó a perfilarse como el vértice de la investigación mundial de la comunicación. Además, otras características que entonces precisaba Marques son: c) La notable y creciente intercomunicación entre los investigadores(as) y proyectos de investigación (además de la concreción de sus publicaciones) como producto de que los investigadores(as) latinoamericanos(as) consideraban su tarea como una empresa subcontinental ligada a la experiencia en el posicionamiento y la transformación política y ética latinoamericana.

Con todo, para José Marques (2000, pp. 22-23), la principal característica que, hasta hoy, ha identificado al conocimiento que genera e identifica a la ELACOM y, por tanto, "a las elaboraciones científicas" que históricamente "han desarrollado los pensadores latinoamericanos" de la comunicación y que ha llegado a plasmar "una singular investigación mestiza representativa de la fisonomía cultural latinoamericana, es el hibridismo teórico y la superposición metodológica”. En la visión de Marques (2000, pp. 22-23), este perfil se caracteriza por varios cruces de tradiciones de investigación y estudio de la comunicación que encontraron su cause natural en la región después de la creación de la CIESPAL -1959- y una vez que se minimizaron las mediaciones gubernamentales y se privilegió el intercambio entre universidades, fundaciones e institutos de investigación; mezclando los paradigmas norteamericanos con los postulados europeos y adaptándolos a las condiciones propias de la sociedad y la cultura latinoamericana, fue posible superar dicotomías entre metodologías cuantitativas y cualitativas, entre búsqueda crítica e indagación administrada.

Para Marques (1999, pp. 4-5), fue así como "construimos una vía latinoamericana para estudiar e interpretar los procesos comunicacionales, anticipándonos tal vez a la superación de los tabúes impuestos por la guerra fría y por las barreras creadas entre Humanidades y Ciencias Sociales...La mezcla distintiva de tales elaboraciones científicas es el hibridismo teórico y la superposición metodológica, que se caracteriza por los cruces de tradiciones europeas, herencias meso-suramericanas (pre y postcolombinas), costumbres africanas, innovaciones de modernas matrices norteamericanas, además de muchas contribuciones introducidas por los distintos grupos étnicos que navegaron por los océanos durante las recientes sagas migratorias internacionales". Sin embargo, tanto en 
la instauración de políticas de comunicación históricamente fieles a los legados de los pioneros de la ELACOM, como en las propias perspectivas de desarrollo de la Escuela Latinoamericana de la Comunicación, para el profesor Marques (2004b, pp. 210-211), existen algunas acciones que implican moverse sobre un guión de luchas que se debate sobre dos frentes simultáneos: la batalla de la ciudadanía y la batalla académica de las universidades.

Es decir, de acuerdo con estudios recientes de Marques (2004b, p. 211), la primera se distingue por poseer un carácter difícil, amplio y polifacético. Se desenvuelve en el ámbito de las sociedades nacionales, justo donde se requieren romper las estructuras ancestrales de la exclusión social como la ignorancia y el silencio. Al eliminar la exclusión comunicacional, según Marques, las sociedades latinoamericanas estarán habilitadas para recorrer el camino de la elevación cultural de sus industrias mediáticas y, consecuentemente, para forjar ciudadanos que hagan de la democracia el instrumento de la coexistencia pacifica y de cooperación civilizada. En el ámbito de la batalla académica, que se desarrolla en las universidades, Marques la caracteriza como un frente específico, sinuoso y resistente, que ha debilitado y conducido, a veces, a la inercia corporativa; su inhibidor central ha sido un elemento ya adelantado arriba, el "sindrome del colonizado". Por "síndrome del colonizado", José Marques (2003b, p.15), entiende el "comportamiento insólito de amnesia histórica" de "renunciar a la tarea de examinar críticamente el referencial teórico acumulado por las generaciones que las precedieron, reproduciendo sin discusión los cuadros cronológicos esbozados en los países que lideran a la producción académica mundial. Por eso mismo, dejan de reconocer el mérito de pensadores que actuaron adelantando la hora, como bien decía el poeta Carlos Drummond de Andrade".

Para superar, además, lo que Marques (2003a, p.209) llama "tendencia del comportamiento aislacionista”, que ha debilitado al campo académico, y rescatar el compromiso histórico con la transformación latinoamericana, Marques propone para debate de la comunidad latinoamericana de Ciencias de la Comunicación aquellas tres líneas de acción concretas que referimos anteriormente: el trabajo sobre la naturaleza del proceso de la comunicación; la autonomía teórica y el fomento a la crítica metodológica y, por último, el rescate del conocimiento empírico, en su triple dimensión: autóctono, mestizo y popular. Al plantear estas líneas de acción, Marques (2004b, pp. 210-211), cree que pueden ayudar a transformar la injusta realidad de déficit cultural y exclusión comunicacional a la que históricamente ha sido sometida Latinoamérica. Para ello, sostiene que se deben superar varias posturas, comenzando "por la superación de la casi atrofia

Escuela Latinoamericana de Comunicación (ELACOM): referente histórico y conquista $\bullet 69$ de la hegemonia en el pensamiento latinoamericano de la comunicación 
al que se auto condenaron nuestras comunidades académicas. Inapetentes para el trabajo cooperativo, ciegas en relación a los proyectos holísticos, temerosas en reconocer el mérito de las generaciones precedentes, avergonzadas ante su propia identidad, confusas delante del pasado, desconfiadas en relación al futuro" (Marques, 2004b, pp. 211).

Sin embargo, se debe reconocer que la batalla académica también va más allá. La disputa académica que se lleva a cabo en algunos centros académicos y de investigación en América Latina muestra claros tintes de cautela e, incluso, resistencias, frente a lo que se ha dado en llamar Escuela Latinoamericana de la Comunicación. Como se recordará, desde 1999, la afinidad en torno a la iniciativa de hablar de ELACOM no ha sido compartida por algunos investigadores destacados del campo académico de la comunicación; por ejemplo, algunos investigadores del Instituto Tecnológico de Estudios Superiores de Occidente (ITESo), ubicado en la Ciudad de Guadalajara, México y a la sazón uno de los centros de enseñanza e investigación de la comunicación más importantes y reconocidos de este país y de Latinoamérica, han sobresalido por entrar en discrepancia pública frente a la iniciativa que lidera Marques. Por un lado, ha sobresalido la voz de Guillermo Orozco (Torrico, 2000, pp. 8-9), impulsor de los estudios cualitativos sobre recepción en México, al poner en duda la iniciativa de hablar de una Escuela como tal, porque "ello conllevaría a algunas homogeneidades de que la comunicología latinoamericana carece”. Además, argumenta y afirma, que si acaso existe una Escuela Latinoamericana de la Comunicación, recién empieza a configurarse pero entorno a la propuesta de Jesús Martín Barbero, quien ha revolucionado los abordajes teórico-metodológicos de los procesos comunicativos a partir de los medios y las mediaciones (Torrico, 2000, pp. 8-9). Por otro lado, ha sido bastante conocida la postura que Raúl Fuentes Navarro ha tomado respecto de la iniciativa y el programa de trabajo de la еLACOM. El Profesor Fuentes, uno de los principales impulsores y estudiosos del campo académico y de la investigación de la comunicación en México y Latinoamérica, sitúa bajo duda el modelo de práctica social que desde 1997 realiza el investigador brasileño José Marques porque éste ubica el presente y el futuro de la investigación brasileńa y latinoamericana bajo la sombra de la institucionalización de la Escuela Latinoamericana de la Comunicación.

Es bastante notorio que las críticas de Fuentes (1999, pp. 52-67) han logrado un único y exclusivo reconocimiento hacia la figura y propuesta de José Marques en virtud de que su propuesta "es exactamente análoga" a la que realizó durante la década de los 50’s y 60’s en Estados Unidos de América el Profesor Wilbur 
Schramm: "El modelo que subyace en los análisis y las propuestas de Marques -y en las acciones colectivas que se ha encargado de liderar- a mi manera de ver es exactamente análogo al que subyacía en la agencia de Wilbur Schramm en los ańos cincuenta y sesenta en Estados Unidos, cuando se constituyó bajo su liderazgo y autoridad el campo académico de la investigación de la comunicación...A pesar de que ahora, como novedad en su larga y reconocida trayectoria, Marques apoya su argumentación en autores como Kuhn y Bourdieu para reconocer el anclaje sociopolítico y la dinámica de tensiones y contradicciones de las estrategias de legitimación en el campo académico, su propuesta se asimila mucho más como una ruptura en el plano ideológico que en cualquier otro. El modelo de práctica social en que Marques ubica el presente y el futuro de la investigación brasileña y latinoamericana de la comunicación no es el que él mismo reconstruye como eje de la que llama la «Escuela Latinoamericana de Investigación de la Comunicación», y que probablemente haya sido formulado y asumido más como «utópico» que como utopístico". (Fuentes, 1999, p. 63).

La propuesta de Fuentes (1999, p.65) se realiza "especialmente en relación con la tensión entre la orientación ideológica y la «cientificidad» implícita en la "comunicología» propugnada por Marques". La proposición de Fuentes (1999, p. 65), se centra básicamente en dos ejes: por un lado, en dar mayor sustancia, vía la teoría, a aquellos conceptos centrales del campo, en definir lo comunicativo y propiciar una anarquía en dichos conceptos, libres de lo que Fuentes llama "toda intromisión institucional"; por otro, de que la investigación de la comunicación en América Latina debe alcanzar una reafirmación ética antes que de una reformulación epistemológica basada en una posición utopística, es decir, en una "evaluación seria, racional y realista de las alternativas históricas". (Fuentes, 1999, p.56).

Tal y como hemos descrito anteriormente, el campo académico de la comunicación reviste formas y estrategias específicas de operación en tanto es un universo que comporta relaciones objetivas, constantes y permanentes, entre las distintas posiciones adquiridas por sus agentes y donde cada cual compromete y define, en su competencia con los demás, una determinada posición dentro del campo. El compromiso y la competencia que define Marques (1999, pp. 1-12), tras el asedio de la conquista por la hegemonía institucional del pensamiento comunicacional latinoamericano, demanda enfrentar y superar algunas posturas, inercias y conductas que comportan algunos investigadores de la comunidad académica de la región. Sobre todo, en aquellos donde se "traduce con mayor nitidez el comportamiento de los investigadores que se encontraron con las

Escuela Latinoamericana de Comunicación (ELACOM): referente histórico y conquista $\bullet 71$ de la hegemonia en el pensamiento latinoamericano de la comunicación 
angustias de la guerra fría". Por ejemplo, entre otras posturas y problemas que Marques (1998, pp. 9-13; 1999, pp. 5-6; 2004, p.15) ha identificado, se pueden destacar las siguientes:

a) La baja autoestima de la comunidad académica latinoamericana, impactada por los cambios de políticas económicas que disminuyen el protagonismo del Estado paternal y atribuyen papeles decisivos al mercado y a la sociedad civil.

b) El proceso de difusión del pensamiento comunicacional latinoamericano es lento y, además, se enfrenta a las barreras que suponen la moda teórica, el comportamiento insólito de la amnesia histórica o el prejuicio de cuantos siguen valorizando exclusivamente las metodologías importadas de los países industrializados.

c) La comunidad académica latinoamericana, por paradójico que parezca, ha relegado a la Escuela Latinoamericana de la Comunicación a una posición secundaria en la mayoría de los cursos de comunicación social en Latinoamérica, sino ausente; ello se ha manifestado en una parte de jóvenes investigadores que ignoran las contribuciones de científicos de la mega región, iluminando y fundamentando sus hipótesis de trabajo exclusivamente en referencias foráneas.

d) La ausencia de una comunidad académica institucionalmente organizada en el área de las ciencias de la comunicación. En los programas de maestría y doctorado que han asumido el desafío de formar exclusivamente recursos humanos destinados a las prácticas comunicacionales (en medios masivos, en empresas o instituciones de la sociedad civil) asientan obstáculos estructurales importantes, como por ejemplo, la falta de disponibilidad de fuentes documentales latinoamericanas y las dificultades para el intercambio y la movilidad científica dentro de la propia mega región.

e) La falta de agilidad de la propia comunidad académica para responder pronto a las cuestiones planteadas por los centros contemporáneos de decisión, localizados en las empresas o en el sector público.

f) La dependencia del mercado, uno de los estigmas heredados del pasado, que impide establecer agendas de investigación que correspondan con las demandas de las industrias culturales.

g) El miedo de convertirse en apéndice de las estructuras estatales ha distanciado también la participación de la comunidad académica latinoamericana de las tomas de decisión sobre las políticas públicas. 
Al constituir el Programa de Doctorado en Comunicación Social en la Universidad Metodista de São Paulo, a partir de 1995, José Marques (1998, pp. 10-11), buscó establecer un conjunto de acciones que pudieran desarrollar estudios comprometidos y que pudieran, a la vez, situar y establecer de manera hegemónica el pensamiento comunicacional latinoamericano. En aras de alcanzar esta meta, Marques ha llevado a cabo durante varios años y, por lo que se puede apreciar en su impresionante producción académica, con una constancia permanente, diversas estrategias y prácticas académicas puntuales. Desde nuestra óptica y desde nuestra particular tarea de búsqueda sobre los orígenes institucionales que han apoyado el programa de trabajo de la ALAIC, se acentúan como acciones y apoyos centrales, las siguientes:

1. Un primer apoyo institucional ha sido la Universidad Metodista de São Paulo, a través de la Facultad de Comunicación Social y el Programa de Doctorado en Comunicación Social (FCS-UMESP). La UMESP surge del Instituto Metodista de Ensino Superior (IMS), el cual se funda en 1970 en el Campus de Rudge Ramos, Municipio de Sao Bernardo do Campo, y está destinado a mantener la Federación de Escuelas Superiores del ABC (conjunto de facultades que ofrecen cursos de pre y postgrado en diversas áreas de conocimiento) del Consejo Federal de Educación de Brasil. De acuerdo con Marques (1996, p.158), de esta iniciativa se constituye, en 1973, la Facultad de Comunicación Social de la Universidad Metodista de Sao Paulo (FCS-UMESP). Las directrices pedagógicas de la FCS-UMESP valoran la formación cultural humana, procurando mantener sintonía con las demandas del mercado laboral de las áreas de su especialización: el periodismo, la radio y televisión, las relaciones públicas, publicidad y propaganda; en la FCS-UMESP se ha procurado mantener un contacto permanente y estrecho con las principales asociaciones de enseñanza e investigación como la International Association for Media and Communications Research (IAMCR), la Federación Latinoamericana de Facultades de Comunicación Social (FELAFACS) o la ALAIC; la FCS-UMESP crea el primer curso de postgrado en 1978 con la Maestría en Comunicación Social y, desde agosto de 1994, el Programa de Doctorado en Comunicación Social. Dentro de los apoyos relevantes que la UMEsp brinda al programa de trabajo anual que suponen los encuentros CELACOM se encuentran los apoyos de trasporte y hospedaje de los profesores visitantes y conferencistas que han recibido tanto la FCS-UMESP como los Coloquios; en este sentido, se destacan, entre otras personalidades relevantes, los nombres de Armand Mattelart, Jorge González, Elizabeth Fox, Juan Díaz Bordenave, Manuel Parés i Maicas, Carmen Gómez Mont, Luis Ramiro Beltrán, Jesús Martín Barbero, etc.

Escuela Latinoamericana de Comunicación (ELACOM): referente histórico y conquista $\bullet 73$ de la hegemonía en el pensamiento latinoamericano de la comunicación 
2. Un segundo apoyo institucional de la mayor relevancia la ha brindado la UNESCO. A partir de 1996, la FCS-UMESP pasa a ser sede de la Cátedra UNESCO de Comunicación para el Desarrollo Regional a través del convenio promovido con la División de Comunicación de la unesco, con sede en París, Francia. La Cátedra unesco de Comunicación en Brasil es supervisada por la Consejería Regional de unEsco en América Latina, con sede en Quito, Ecuador, y afiliada a la World Network of unesco Communication Chairs (оRBICOM), cuya sede se encuentra en Montreal, Canadá. Como anteriormente se anotó, la Organización de las Naciones Unidas para la Educación, la Ciencia y la Cultura (UNESCO) fue creada el 16 de noviembre de 1945 por la Organización de las Naciones Unidas (ONU) durante la conferencia promovida para tal caso en Londres; casi medio siglo después de su fundación, se crea en 1989 la primera Cátedra unEsco de Comunicación a nivel mundial en la Facultad de Ciencias de la Comunicación de la Universidad Autónoma de Barcelona, Campus Bellaterra, bajo la destacada y solidaria coordinación del profesor catalán Dr. Manuel Parés y Maicas. Entre los apoyos importantes que la Cátedra unesco de Comunicación en Brasil destina al programa de trabajo anual que implican los encuentros CELACOM se encuentran: a) La financiación parcial para la realización de los encuentros CELACOM; b) La financiación para realizar investigación sobre dos ejes de acción particulares: la construcción de diagnósticos situacionales y la elaboración de perfiles biográficos de instituciones e investigadores paradigmáticos en la conformación de la Escuela Latinoamericana de la Comunicación y, c) La financiación para publicación de los libros y artículos que proyectan a la ELACOM en la comunidad académica de la región, incluyéndose aquí la financiación para publicar las contribuciones anuales recibidas en los CЕLACOM bajo el nombre de "Serie Análisis de la Escuela Latinoamericana de la Comunicación".

3. Un tercer apoyo institucional concreto que otorga la UMESP y que consideramos de alta relevancia para alcanzar la meta de situar y establecer de manera hegemónica el pensamiento comunicacional latinoamericano, fue la adquisición de un gran acervo documental y bibliográfico que rescata la memoria de las historias colectivas e individuales de buena parte de los trabajos que incorporaban el pensamiento comunicacional Latinoamericano. Dicha memoria se plasma en un amplio repertorio documental formado por 10.000 volúmenes, correspondientes la adquisiciones bibliográficas y hemerográficas hechas por el profesor José Marques durante las décadas de 1960 y 1970, años que fueron determinantes para la formación académica de Marques y, como ya sabemos, fueron décadas también definitorias en la formación del campo académico de 
la comunicación en América Latina. Este acervo, que durante el primer trienio fortalecería el patrimonio documental de los cursos de Doctorado en Comunicación Social de la UMESP, rápidamente fue haciéndose fuente de referencia para los estudiantes, docentes e investigadores de la casa. Desde 1998, el Acervo fue procesado para su incorporación física al depósito de la Biblioteca Central de la uMESP y, desde ese año, paso también a formar parte integrante de lo que en la Cátedra unesco de Comunicación de Brasil denomina como el Acervo de la Escuela Latinoamericana de Comunicación.

4. Sin embargo, tal vez el apoyo institucional más importante y trascendental que tanto la UMESP como la Cátedra UNESCo han desarrollado hasta ahora en favor de implantar de manera hegemónica el pensamiento comunicacional latinoamericano, a través del programa de trabajo de la Escuela Latinoamericana de la Comunicación, han sido los Coloquios Internacionales de Estudios sobre la Escuela Latinoamericana de Comunicación (СELACOM). De acuerdo con Marques (2004), los apoyos y recursos que dedica la Universidad Metodista de Sao Paulo para la financiación y realización de los CELACOM viene dado por el material de información y divulgación que se ofrecen en los encuentros como por los costes de viaje y hospedaje de los invitados y ponentes especiales. Por otro lado, de la Cátedra unEsCo se reciben los fondos que garantizan las publicaciones de cada Coloquio anual y ello representa lo que en la Cátedra unesco Brasil se llama "Serie de Análisis de la Escuela Latinoamericana de la Comunicación”. Los Coloquios Internacionales de la ELACOM se han caracterizado, según la visión de su director, por ser espacios destinados a hacer historia, con la participación física y directa de aquellos hombres, mujeres e instituciones que han sido y son protagonistas en la conformación de la Escuela Latinoamericana de la Comunicación (Marques, 1998, pp. 1-9). Los espacios de los CELACOM, permiten al público asistente, tradicionalmente formado por investigadores, profesores y estudiantes, interaccionar directamente con los investigadores invitados y estudiados. De este modo se ha logrado establecer una relación inédita, donde los mitos son sustituidos por "verdades", tanto en la obra como en sus creadores. Esta relación tiene, pues, el objetivo de promover el debate, el conocimiento y la ampliación de las ideas comunicacionales, facilitar su entendimiento y la asimilación de sus teorías y conceptos generados (Marques, 1998, pp. 12-13).

La difusión internacional de la ELACOM inicio en marzo de 1997 con el estudio de las ideas comunicacionales del investigador boliviano Luís Ramiro Beltrán. La selección de Beltrán entre el destacado y selecto grupo de pioneros

Escuela Latinoamericana de Comunicación (ELACOM): referente histórico y conquista $\bullet 75$ de la hegemonia en el pensamiento latinoamericano de la comunicación 
de la llamada Escuela Latinoamericana de la Comunicación obedeció, según Marques (1998, pp. 12-13) no sólo a su "estatura intelectual como padre de las políticas de comunicación en la región sino, y, sobre todo, por su dimensión humana, traducida en su postura ética, científica y ciudadana, sirviendo como referencial para las nuevas generaciones de comunicadores y comunicólogos. Luís Ramiro Beltrán simboliza justamente aquel paradigma de científico de la comunicación que puede ayudar a nuestra comunidad a construir y fortalecer su identidad académica". De este primer encuentro CELACOM, realizado en el Campus UMESP de la Ciudad de Sáo Bernardo do Campo (São Paulo, Brasil), se editó el libro que abre nuestro análisis sobre la ЕLACOM: $A$ Trajetória Comunicacional de Luiz Ramiro Beltrán. El volumen, que consta de 168 páginas, fue organizado por los Profesores José Marques y Juçara Gorski Brittes (1998, pp. 9-168). La publicación reúne catorce contribuciones de amigos y discípulos de Luís Ramiro Beltrán como de varios investigadores de Brasil, Estados Unidos y América Latina, especializados en políticas de comunicación para el desarrollo y en las iniciativas pioneras de la legitimación académica de la Escuela Latinoamericana de la Comunicación.

El II CELACOM llevado a cabo en el Campus de la umesp durante el mes de septiembre de 1998, tuvo como temática de estudio el recorrido intelectual de Jesús Martín Barbero. La obra de Martín Barbero es actualmente una de las pocas obras nativas de la región que son ampliamente estudiadas en la mayoría de las universidades latinoamericanas. La influencia de su trabajo puede ser observada en las diversas sociedades científicas latinoamericanas del área de la comunicación, la sociología y la antropología; en el discurso de apertura del II CELACOM, el profesor Marques, dijo que "las historias de vida que la Cátedra UNESCO han promovido asumen un carácter público para que el rescate de la memoria sea también público, y es seguro que la obra de Jesús Martín Barbero es la historia viva de una trayectoria intelectual de grandes contribuciones para los estudios de comunicación y difusión del pensamiento latinoamericano". (Marques, 1998, pp. 1-9). El resultado del II CELACOM fue editado por Marques y Rocha Dias (1999, pp. 9-213) bajo el título Comunicação, Cultura, Mediaçóes. O Percurso Intelectual de Jesús Martín-Barbero. La publicación reúne doce contribuciones de investigadores especializados sobre el recorrido intelectual, docente y de investigación por el que ha transitado Jesús Martín Barbero durante sus 69 años de vida.

El III Coloquio CELACOM de 1999, que ocurrió entre el 5 y 7 de mayo, tuvo como temática central la "Génesis del Pensamiento Comunicacional Latino- 
americano. El protagonismo de las instituciones pioneras: CIESPAL, ICINFORM e ININCO". Con este tema, la Cátedra UNESCO busco evidenciar la difusión y el desarrollo de las ideas comunicacionales de la Escuela Latinoamericana de la Comunicación a través del proceso de creación y consolidación que tuvieron estas instituciones. Además, aquí se busco rescatar el desarrollo histórico de tres instituciones académicas paradigmáticas, particularmente responsables de la construcción de matrices teóricas de la comunicación en procesos de desarrollo. De este tercer encuentro Celacom, se editó el libro Gênese do Pensamento Comunicacional Latino-Americano. O Protagonismo das Instituiçôes Pioneiras: CIESPAL, ICINFORM, ININCO. El volumen fue organizado por los Profesores José Marques y Maria Cristina Gobbi (2000, pp. 9-304). La publicación reúne a dieciocho contribuciones especializadas sobre el papel de CIESPAL en la difusión de las ideas comunicacionales hegemónicas en la producción de las matrices latinoamericanas, el diálogo popular masivo de ICINFORM y, por último, el paradigma de la investigación-denuncia que desarrolla el ININCO.

El IV Coloquio Internacional de Estudios sobre la Escuela Latinoamericana de Comunicación se realizó en mayo del 2000 en el Campus Rudge Ramos de la uMESP. La temática del encuentro fue dedicada a las "Contribuciones Brasileras de la Comunicación”. De acuerdo con Marques (Marques, Gobbi y Santos, 2001, p.29), fue a partir de considerar las singularidades de la contribución brasileña al pensamiento comunicacional latinoamericano que la cátedra UNESCO tomó la decisión de escoger para la agenda de trabajo del CELACOM 2000 a tres autores emblemáticos: Décio Pignatari, Muniz Sodré y Sérgio Caparelli; de las contribuciones recibidas, los Profesores José Marques, Maria Cristina Gobbi e Marli dos Santos (2000, pp. 9-126) editaron el libro Contribuiçôes Brasileiras ao Pensamento Comunicacional Latino-Americano: Décio Pignatari, Muniz Sodré e Sérgio Capparelli. Esta publicación reúne doce contribuciones especializadas sobre la contribución realizada por estos tres autores emblemáticos al pensamiento comunicacional latinoamericano.

El V CELACOM se llevo a cabo en mayo de 2001 y su temática se tituló "Marxismo y Cristianismo, Matrices de las Ideas Comunicacionales en América Latina"; de acuerdo con Marques (2002, pp. 10-12) este Coloquio 2001 trata de hacer un balance de dos de las corrientes que constituyen un manantial simbólico en la conformación del pensamiento comunicacional latinoamericano y de recorrer el camino cultural en el cual se proyectó la ELACOM, cuya generación pionera fue educada siguiendo los parámetros ideológicos del cristianismo sin mostrar desprecio por los signos desafiadores del marxismo. Como producto

Escuela Latinoamericana de Comunicación (ELACOM): referente histórico y conquista $\bullet 77$ de la hegemonia en el pensamiento latinoamericano de la comunicación 
de este V Coloquio, se editó el libro Matrices Comunicacionais Latino-Americanas: Marxismo e Cristianismo, a cargo de los Profesores José Marques, Maria Cristina Gobbi y Waldemar Luiz Kunsch (2002, pp. 9-126). La publicación reúne doce contribuciones especializadas sobre las aportaciones del marxismo y el cristianismo al impulso de las ideas comunicacionales latinoamericanas como a las estrategias de las políticas de comunicación de la región.

El VI CeLACOM se realizó en la Ciudad de Adamantina, en São Paulo, Brasil, la primera semana de octubre de 2002. El tema central del evento fue "la participación de la mujer en los estudios comunicacionales latinoamericanos". De acuerdo con Marques (2003, pp. 9-10), la sexta jornada del CELACOM se llevó a cabo para entender cómo se da la participación de la mujer en la investigación de la comunicación en América Latina y tratar de evocar en las nuevas generaciones de investigadores(as) la discusión sobre los procesos de inserción femenina en la comunidad académica de la mega región. El VI Coloquio trajo como fruto el libro titulado Comunicação Latino-Americana. O Protagonismo Femenino; este documento, de 255 páginas, fue editado por los Profesores José Marques, Maria Cristina Gobbi y Sérgio Barbosa (2003, pp. 9-255). Aquí se apiñan dieciocho contribuciones especializadas sobre la participación de las mujeres en la producción de conocimiento, en la agenda mediática y el papel que han desarrollado como profesionales del campo de la comunicación en el contexto latinoamericano.

El VII celacom se llevó a cabo en el Campus umesp de la Ciudad de São Bernardo do Campo, en São Paulo, Brasil, entre el 26 y 28 de mayo de 2003. La agenda del VII Coloquio fue marcada por el pensamiento crítico y los impactos en la vida de la sociedad latinoamericana. El propósito fue, de acuerdo con Marques (2004, pp. 15-18) "hacer un balance del camino recorrido, esbozando al mismo tiempo las rutas por las cuales debemos transitar de ahora en adelante"; el tema central del programa tuvo tres ejes protagónicos: 1) El pensamiento crítico en la investigación comunicacional de América Latina: de la investigación denuncia a la investigación acción; 2) El pensamiento crítico en la construcción de alternativas mediáticas Latinoamericanas: conquistas y fracasos y, 3) El Grupo Comunicacional de São Bernardo: el pensamiento crítico o el pragmatismo utópico. De las contribuciones recibidas al encuentro, los Profesores Marques y Maria Cristina Gobbi (2004, pp. 9-338), organizaron la edición del volumen titulado Pensamento Comunicacional Latino-Americano. Da Pesquisa-Denúncia Ao Pragmatismo Utópico. Esta publicación, que es la más corpulenta de la "Serie Análisis de la Escuela Latinoamericana de la Comuni- 
cación”, reúne a veinticuatro contribuciones especializadas sobre los tres ejes de la temática.

El último evento realizado y divulgado hasta hoy en día sobre la Escuela Latinoamericana de la Comunicación es el VIII Coloquio Internacional de Estudios sobre la ELACOM. Se llevó a cabo en el Campus Rudge Ramos de la umesP (São Paulo, Brasil), entre el 29 y 31 de marzo de 2004. La agenda del VIII Coloquio estuvo marcada por tres ejes centrales de análisis y debate: a). La sociedad del conocimiento: la democratización del conocimiento; b). Conflictos y coexistencia con las redes telemáticas; y, c). El derecho de la propiedad intelectual: repercusiones en las industrias de contenido. De las contribuciones recibidas al VIII CELACOM, los Profesores José Marques, Paulo Tarsitano, Luciano Sathler y Maria Cristina Gobbi (2005, pp. 9-250), organizaron la edición del volumen titulado Sociedade do Conhecimento. Aportes Latino-americanos. El libro reúne a quince contribuciones especializadas sobre los tres ejes de la temática.

En resumen, la obra de la "Serie Análisis de la Escuela Latinoamericana de la Comunicación", comprende un total de ocho volúmenes, uno por cada CELACOM realizado entre 1997 y el 2004; las publicaciones analizadas salen a la luz entre 1998 y 2005, es decir, las publicaciones aparecen un año después de celebrado el Coloquio respectivo. Por los ocho títulos publicados en el periodo, se tiene ya claro la diversidad de temas, objetos y preocupaciones que se tienen bajo análisis de estudio: desde el recorrido intelectual y las contribuciones al pensamiento comunicacional latinoamericano que han forjado personalidades de la talla de Luís Ramiro Beltrán, Jesús Martín Barbero, Antonio Pasquali, Luiz Beltrão, Sérgio Capparelli, Muniz Sodré y Décio Piganatari; hasta las aportaciones que el marxismo, el cristianismo, la participación de la mujer y el pensamiento crítico han aportado en la conformación y el impulso de las ideas comunicacionales latinoamericanas, sin dejar de lado, además, el estudio de aquellas instituciones pioneras que desarrollaron las ideas comunicacionales de la Escuela Latinoamericana de la Comunicación como CIESPAL, ICINFORM e InINCO. En los ocho títulos publicados se suman un total de 148 contribuciones o trabajos de investigación, lo que representa un promedio de dieciocho ponencias por libro anual publicado. Sin entrar a analizar aquí los problemas o virtudes que ello conlleva, podríamos afirmar que la "Serie Análisis de la Escuela Latinoamericana de la Comunicación” es una obra fundamentalmente escrita en lengua lusitana, pues del total de contribuciones publicadas en los ocho volúmenes, el $81 \%$ son textos escritos en portugués y el $19 \%$ restante en castellano.

Escuela Latinoamericana de Comunicación (ELACOM): referente histórico y conquista $\bullet 79$ de la hegemonia en el pensamiento latinoamericano de la comunicación 


\section{Conclusiones}

Hasta aquí se han desarrollado algunas líneas y guías de análisis que pueden considerarse abiertas para una exploración más profunda y rica en la identificación de los contextos históricos y las características y tesis que le son propias a la construcción de la idea que encarna la ELACOM. Hemos tratado de abrir algunas líneas y guías de proximidad histórica desde la iniciativa y la perspectiva conceptual y discursiva que le ha suministrado no solamente su procedencia y razón institucional, sino, además, en tratar de conocer el porqué de la ubicación en debate del programa de la Escuela Latinoamericana de la Comunicación. Se concluye que es en Brasil y en el Programa de Doctorado en Comunicación Social de la Universidad Metodista de Sáo Paulo, el mismo que incorpora la cátedra unEsco-Comunicación para el Desarrollo Regional de Brasil, donde surge, se auspicia y fomenta la tesis de la existencia de la ELACOM, inspiración creada por José Marques. El apoyo institucional más importante y trascendental que tanto la UMESP como la Cátedra UNESCo han desarrollado hasta ahora en favor de implantar de manera hegemónica el pensamiento comunicacional latinoamericano, a través del programa de trabajo de la Escuela Latinoamericana de la Comunicación, han sido los apoyos necesarios para realizar los CELACOM. Uno de estos apoyos es la publicación de la obra Serie Análisis de la Escuela Latinoamericana de la Comunicación, que comprende la publicación de las investigaciones presentadas en cada CELACOM anual. Sus primeras ocho publicaciones salen a la luz entre 1998 y 2005. Por los ocho títulos publicados en el periodo, se tiene ya claro que los discursos textuales del capital científico que participa en la obra ELACOM se perfila a priorizar los y las protagonistas, los procesos y las condiciones históricas que marcan los distintos periodos y rasgos de identidad en el Pensamiento Latinoamericano de la Comunicación (PLC).

Ha quedado claro que la iniciativa ELACOM es una iniciativa institucional con un paso ascendente tras la búsqueda de la identidad latinoamericana de la comunicación. Que las prácticas científicas del colectivo que ahí publica definitivamente pueden incidir en volcar el pensamiento latinoamericano de la comunicación como un paradigma dominante en los estudios de la comunicación en la región, aunque definitivamente no sea esta la única iniciativa. Se concluye que la iniciativa ELACOM es el producto de una forma específica de trabajo y de intereses que desarrolla en gran medida su principal impulsor, José Marques. En tanto un agente dominante dentro de la iniciativa y el proyecto institucional de la ELACOM, José Marques ha adoptado, con el apoyo de un destacado grupo de 
protagonistas, un conjunto de prácticas y estrategias de conservación del PLC tendientes a perpetuar una historia y un cierto orden científico establecido del cual él mismo es una parte importante e interesada. Por ello, se debe esperar que su participación en la obra ELACOM nunca aparezca como desinteresada. Su presencia en la obra conjunta, tal y como lo constata el reconocimiento de citación en esta, es por demás relevante y decidida. La aspiración tan alta que define a Marques tras situar y establecer de manera hegemónica el pensamiento comunicacional latinoamericano a través de la obra ELACOM depende (y seguirá dependiendo) de un mayor consenso e inversión en el capital científico, sobre todo del propio reconocimiento de los pares competidores que participan y que sigan participando en la iniciativa. El conjunto de recursos científicos heredados del PLC que existen hoy en estado objetivado, bajo la forma de instrumentos, de obras y de instituciones, así como en estado incorporado, bajo la forma de habitus científico, merecen sin duda un mayor esfuerzo para materializar y ampliar la iniciativa de Marques. No obstante debe considerarse el más amplio consenso de participación de la comunidad académica latinoamericana de la comunicación y garantizar la promoción dentro del conjunto de instituciones de educación superior de América Latina, para asegurar desde ahí su producción, reproducción y circulación en el sistema de enseñanza.

\section{BibLIOGRAFÍA}

Beltrán, L. R. (1974). Communication research in Latin America: the blindfolded inquiry?, documento de la Conference on the Contribution of the Mass Media to the Development of Consciousness in a Changing World, Leipzig, República Democrática de Alemania, pp. 1-23.

. (1978). Premisas, objetos y métodos foráneos en la investigación sobre comunicación en Latinoamérica, Órbita, núm. 22, pp. 3-35.

(1978a). Estado y perspectivas de la investigación en comunicación en América Latina. ITESO. Textos Escogidos. México.

(1982). No renunciemos jamás a la utopía, entrevista de Patricia Anzola. Chasqui, No. 3, CIESPAL, Quito, p.6-13.

(1999). Las politicas nacionales de comunicación (PNC) son hoy -en el mundo globalizado e internetizado- más necesarias que nunca, Pensamiento comunicacional latinoamericano, UMESP/UNESCO. Núm. 1, pp. 1-7. . (2004). Comunicación y desarrollos democráticos en Latinoamérica. Comunicación, No. 126, Centro Gumilla. Caracas, pp. 79-94.

Escuela Latinoamericana de Comunicación (ELACOM): referente histórico y conquista $\bullet 81$ de la hegemonía en el pensamiento latinoamericano de la comunicación 
Fadul, A. (1979). Teoria e prática no ensino da comunicação. En, Marques, J; Fadul, A; y Lins da Silva, C. (1979). Ideología y poder no ensino de comunicação. São Paulo. Cortes y Moraes, pp. 50-57.

Gobbi, M. C. (2001). Grandes nomes da comunicação: José Marques. Recife, Brasil. UNICAP - CENTRO, pp. 7-323.

Fuentes, R. (1999). La investigación de la comunicación en América Latina: condiciones y perspectivas para el siglo XXI, Diálogos de la Comunicación, núm. 56, Lima, Perú, Federación Latinoamericana de Facultades de Comunicación, pp. 54-67.

León, G. (2005). Institucionalización del Campo Académico de la Comunicación en América Latina. Un Acercamiento a las Caracteristicas Estructurales de la Escuela Latinoamericana de la Comunicación. Trabajo de Investigación doctoral. Universidad Autónoma de Barcelona. 1501002895/TR344; pp: 7-324.

Marques, J. (1978). Comunicação, modernização e difusão de inovações no Brasil, Petrópolis. Vozes, pp. 7-252.

- (1999). Desarrollo y Perspectivas de la Escuela Latinoamericana de Comunicación. Ponencia presentada en el I Encuentro Nacional de Investigadores de la Comunicación de Bolivia y el I Seminario Latinoamericano de Investigadores en Comunicación. Cochabamba, Bolivia; noviembre de 1999. pp. 1-12.

(1999a). A Comunicade Académica das Ciencias da Comunicação: Revisao Crítica da Experiencia Brasileira como Paradigma para o Fortalecimento da Comunidade Latino-Americana. Ponencia presentada en la conferencia inaugural de X Encuentro AMIC. La Trinidad, México. Abril de 1999. pp. $1-13$.

(1999b). Prefacio. En, Marques, J. y Rocha, P. (1999). Comunicação, cultura, mediaçôes. O percurso intelectual de Jesús Martín-Barbero. São Bernardo do Campo, São Paulo, Brasil, UNESCO - UMESP. II CELACOM, pp. 9-12.

(2004). Prefacio. En, Marques, J.; Gobbi, M. (2004) Pensamento Comunicacional Latino-Americano. São Bernardo do Campo, São Paulo, Brasil. UNESCO - UMESP. VII CELACOM, pp. 15-18.

. (2004a). Los tiempos heroicos. La formación de la comunidad latinoamericana de la comunicación. Revista Latinoamericana de Ciencias de la Comunicación. ALAIC. São Paulo, Brasil, pp. 10-21.

. (2004b). A esfinge mediática. São Paulo. Paulus, pp. 11-335.

Marques, J; Fadul, A; y Lins da Silva, C. (1979). Ideología y poder no ensino de comunicação. São Paulo. Cortes y Moraes, pp. 9-168. 
Marques, J. y Gorski, J. (1998). A trajetória comunicacional de Luiz Ramiro Beltrán, Sao Paulo, UNESCO - UMESP. I CELACOM, pp. 9-168.

Marques, J. y Rocha, P. (1999). Comunicação, cultura, mediaçóes. O percurso intelectual de Jesús Martín-Barbero. São Bernardo do Campo, São Paulo, Brasil, UNESCO - UMESP. II CELACOM, pp. 9-231.

Marques, J. y Gobbi, M. (2000). Gênese do pensamento comunicacional latinoamericano: o protagonismo das instituiçôes pioneiras- CIESPAL, ICINFORM, ININCO. São Bernardo do Campo, São Paulo, Brasil. UNESCO - UMESP. III CELACOM, pp. 9-304.

Marques, J; Gobbi, M; y Dos Santos, M. (2001). Contribuiçóes brasileiras ao pensamento comunicacional latino-americano: Décio Pignatari Muniz Sodré e Sérgio Capparelli. São Bernardo do Campo, São Paulo, Brasil. UNESCO - UMESP. IV CELACOM, pp. 9-126.

Marques, J; Gobbi, M. y Kunsch, L. (2002). Matrizes comunicacionais latinoamericanas - marxismo e cristianismo. São Bernardo do Campo, São Paulo, Brasil. UNESCO - UMESP. V CELACOM, pp. 9-271.

Marques, J; Gobbi, M. y Barbosa, S. (2003). Comunicaçâo latino-americana. O protagonismo femenino. São Bernardo do Campo, São Paulo, Brasil. UNESCO - UMESP - FAI. VI CELACOM, pp. 9-255.

Marques, J. y Gobbi, M. (2004). Pensamento Comunicacional Latino-Americano. São Bernardo do Campo, São Paulo, Brasil. UNESCO - UMESP. VII CELACOM, pp. 9-338.

Marques, J; Tarsitiano, P; Sathler, L; y Gobbi, M. (2005). Sociedade do Conhecimento. Aportes latino-americanos. São Bernardo do Campo, São Paulo, Brasil. UNESCO - UMESP. VIII CELACOM, pp. 9-250.

Nóbrega, M. (2000). "Icinform, uma experiencia pioneira". En, Marques, J. y Gobbi, M. Gênese do pensamento comunicacional latino-americano: o protagonismo das instituiçôes pioneiras- CIESPAL, ICINFORM, ININCO. São Bernardo do Campo, São Paulo, Brasil. UNESCO - UMESP. III CELACOM, pp. 157-166.

Torrico, E. (2000). Eventos: Cochabamba, sede de la más grande reunión académica sobre comunicación en Latinoamérica, Pensamiento Comunicacional Latinoamericano, núm. 2, vol. 1, Universidad Metodista de San Paulo, pp. 8-9.

Escuela Latinoamericana de Comunicación (ELACOM): referente histórico y conquista $\bullet 83$ de la hegemonia en el pensamiento latinoamericano de la comunicación 